\title{
Amorphous silica nanoparticles induce tumorigenesis via regulating ATP5H/SOD1-related oxidative stress oxidative phosphorylation and EIF4G2/PABPC1-associated translational initiation
}

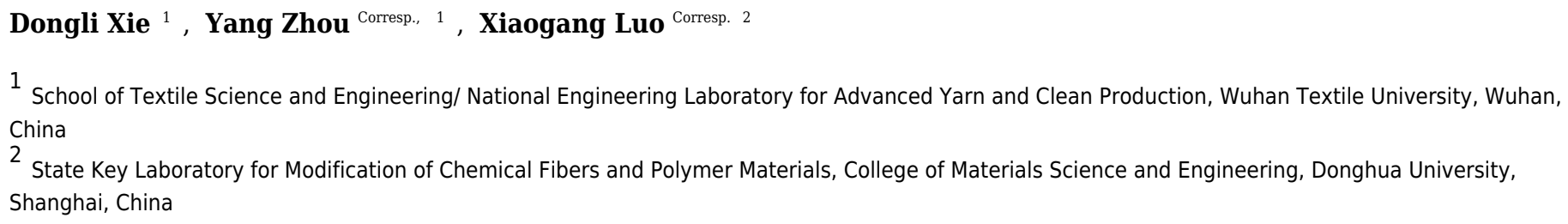

Background: Recent studies indicate amorphous silica nanoparticles (SiNPs), one of the widely applied nanomaterials, have potential toxicity on human and induce cell malignant transformation. However, the carcinogenic mechanisms remains not well understood. This study was to investigate the underlying toxic mechanisms of amorphous SiNPs on human lung epithelial cells model by using microarray data.

Methods: Microarray dataset GSE82062 was collected from Gene Expression Omnibus database, including three repeats of Beas-2B exposed to amorphous SiNPs for 40 passages and three repeats of passage-matched control Beas-2B cells. Differentially expressed genes (DEGs) were identified using LIMMA method. Protein-protein interaction (PPI) network was constructed using data from STRING database followed by module analysis. miRwalk2 database was used to predict the underlying target genes of differentially miRNAs. Function enrichment analysis was performed using DAVID tool.

Results: A total of 323 genes were identified as DEGs, including 280 downregulated (containing 12 premiRNAs) and 43 upregulated genes (containing 29 pre-miRNAs). Function enrichment indicated these genes were involved in translational initiation (i.e. EIF4G2, PABPC1), response to reactive oxygen species (i.e. SOD1) and Oxidative phosphorylation (i.e. ATP5H). PABPC1 (degree = 15), ATP5H (degree = 11) and SOD1 (degree $=8$ )] were proved to be hub genes after PPI-module analyses. ATP5H/SOD1 and EIF4G2/PABPC1 were overlapped with the target genes of differentially expressed pre-miR-3648/572/661 and pre-miR-4521.

Conclusions: Amorphous SiNPs may induce tumorigenesis via influencing ATP5H/SOD1-related oxidative stress oxidative phosphorylation and EIF4G2/PABPC1-associated translational initiation which may be regulated by miR-3648/572/661 and miR-4521, respectively. 
1 Amorphous silica nanoparticles induce tumorigenesis via regulating ATP5H/SOD1-related

2 oxidative stress oxidative phosphorylation and EIF4G2/PABPC1-associated translational

3 initiation

4 Running title: mechanisms of SiNP-induced tumorigenesis

5 Dongli Xie ${ }^{1}$, Yang Zhou ${ }^{1 *}$, Xiaogang Luo ${ }^{2 *}$

$6{ }^{1}$ School of Textile Science and Engineering/ National Engineering Laboratory for Advanced

7 Yarn and Clean Production, Wuhan Textile University, Wuhan 430200, China.

$8{ }^{2}$ State Key Laboratory for Modification of Chemical Fibers and Polymer Materials, College of

9 Materials Science and Engineering, Donghua University, Shanghai 201620, China.

10 Correspondence to: Yang Zhou and Xiaogang Luo.

11 Yang Zhou

12 Correspondence address: School of Textile Science and Engineering/ National Engineering

13 Laboratory for Advanced Yarn and Clean Production, Wuhan Textile University, Wuhan 430200,

14 China.

15 E-mails: yzhou@wtu.edu.cn; Tel: +86-027-59367469.

Xiaogang Luo

Correspondence address: State Key Laboratory for Modification of Chemical Fibers and

Polymer Materials, College of Materials Science and Engineering, Donghua University,

20 Shanghai 201620, China.

21 E-mails: luoxiaogang@dhu.edu.cn; Tel: +86- 021-67792857. 


\section{Abstract}

24 Background: Recent studies indicate amorphous silica nanoparticles (SiNPs), one of the widely applied nanomaterials, have potential toxicity on human and induce cell malignant transformation. However, the carcinogenic mechanisms remains not well understood. This study was to investigate the underlying toxic mechanisms of amorphous SiNPs on human lung epithelial cells model by using microarray data.

Methods: Microarray dataset GSE82062 was collected from Gene Expression Omnibus database, including three repeats of Beas-2B exposed to amorphous SiNPs for 40 passages and three repeats of passage-matched control Beas-2B cells. Differentially expressed genes (DEGs) were identified using LIMMA method. Protein-protein interaction (PPI) network was constructed using data from STRING database followed by module analysis. miRwalk2 database was used to predict the underlying target genes of differentially miRNAs. Function enrichment analysis was performed using DAVID tool.

Results: A total of 323 genes were identified as DEGs, including 280 downregulated (containing 12 pre-miRNAs) and 43 upregulated genes (containing 29 pre-miRNAs). Function enrichment indicated these genes were involved in translational initiation (i.e. EIF4G2, PABPC1), response to reactive oxygen species (i.e. SOD1) and Oxidative phosphorylation (i.e. ATP5H). PABPC1 $($ degree $=15)$, ATP5H $($ degree $=11)$ and SOD1 $($ degree $=8)]$ were proved to be hub genes after PPI-module analyses. ATP5H/SOD1 and EIF4G2/PABPC1 were overlapped with the target genes of differentially expressed pre-miR-3648/572/661 and pre-miR-4521.

Conclusions: Amorphous SiNPs may induce tumorigenesis via influencing ATP5H/SOD1- 
44 related oxidative stress oxidative phosphorylation and EIF4G2/PABPC1-associated translational

45 initiation which may be regulated by miR-3648/572/661 and miR-4521, respectively.

46

47

48

49

50

51

52

53

54

55

56

57

58

59

60

61

62

63

64 Introduction 
65 Nanomaterials refer to those with sizes ranging from 1 to $100 \mathrm{~nm}$ in at least one dimension.

66 Nanosized particles possess a number of superior physicochemical properties compared with the

67 same material fabricated in a conventional manner, including high thermal and chemical stability,

68 hydrophobicity, heat and electrical insulation, resistance to oxidation, good biocompatibility and

69 minimal immunogenicity, which make them as attractive and promising candidates for a wide

70 range of advanced applications (Liang et al. 2008; Peng et al. 2014). Silica is the most frequently

71 used material to create nanoparticles (NPs) due to its higher abundance on earth and thus a

72 relatively low-cost for preparation. It is estimated that approximately one million tons of

73 synthetic amorphous silica nanoparticles (SiNPs) may be produced per year worldwide to act as

74 additives to cosmetics, drug tablets, paints, varnishes, food or deliveries for gene, protein and

75 drugs (Fruijtier-Pölloth 2012). The widespread application of amorphous SiNPs leads to its

76 frequent exposure for human and therefore its safety is the highly concerned issue. Although

77 amorphous SiNPs are suggested not to have carcinogenicity to humans according to the

78 classification of International Agency for Research of Cancer (Group 3), recently published

79 studies proposed long-term exposure of amorphous SiNPs may induce cell malignant

80 transformation and tumorigenesis (Fontana et al. 2017; Guo et al. 2017). Hereby, how to early

81 diagnose and prevent the development of amorphous SiNPs-induced cancer may be underlying

82 challenges that need to be solved. This resulted in the requirements for understanding the

83 molecular mechanisms of the tumor-promoting effects.

84 In a recent study, Guo et al. used a microarray analysis to investigate the genes significantly

85 changed by amorphous SiNPs in human lung epithelial cells and found amorphous SiNPs may 
86

87

trigger tumorigenesis by influencing 821 significant differentially expressed genes (DEGs) (5 upregulated and 816 downregulated) to regulate oxidative stress, oxidative phosphorylation, DNA damage, p53 and MAPK signaling pathways (Guo et al. 2017). However, the related mechanism leading to the development of cancer by amorphous SiNPs still remains unclear. In present study, we aim to re-analyze the microarray data established by Guo et al. (Guo et al. 2017) via different bioinformatic approaches: the DEGs were identified by the Linear Models for Microarray data (LIMMA) method, but not random variance model; Compared with study of Guo et al. (Guo et al. 2017), a strict threshold was used for screening DEGs ( $\operatorname{logFC}$ (fold change) $\mid>2 \& \mathrm{p}<0.05$ vs FC $>2 \& \mathrm{p}<0.05$ ), which may be beneficial to obtain more crucial and verifiable genes associated with amorphous SiNPs; the whole protein-protein interaction (PPI) network for all DEGs were established, but not signal-net analysis network PPI; In addition, miRNA-target genes interaction network was also analyzed to explore the regulatory mechanisms of DEGs and then screen key upstream targets for amorphous SiNPs, which was not performed previously.

\section{Materials \& Methods}

\section{Microarray data}

The microarray data were extracted from the Gene Expression Omnibus (GEO) database (http://www.ncbi.nlm.nih.gov/geo/) under accession number GSE82062 (Guo et al. 2017), in which three repeats of human lung epithelial cells, Beas-2B continuously exposed to $5 \mu \mathrm{g} / \mathrm{mL}$ amorphous SiNPs for 40 passages (BeasSiNPs-P40 group; Supplemental Information 1.1, 1.2, 1.3) and three repeats of passage-matched control Beas-2B cells (Beas-P40 group) 
107 (Supplemental Information 1.4, 1.5, 1.6) were available for the analysis.

108

109

110

111

112

113

114

115

116

117

118

119

120

121

122

123

124

125

126

127

\section{Data normalization and DEGs identification}

The raw data (CEL files) of GSE82062 were downloaded from the Affymetrix Human

Transcriptome Array 2.0 platform GPL17586. The raw data were preprocessed, backgroundcorrected and summarized using Robust Multichip Average (RMA) algorithm (Irizarry et al. 2003) in the 'affy' package of Bioconductor R (v3.4.1;

http://www.bioconductor.org/packages/release/bioc/html/affy.html). The DEGs between BeasSiNPs-P40 and Beas-P40 groups were identified using the Linear Models for Microarray data (LIMMA) method (Ritchie et al. 2015) in the Bioconductor R package (v3.4.1; http://www.bioconductor.org/packages/release/bioc/html/limma.html). The $\mathrm{t}$ test was used to identify the p-value and FC was calculated. Genes were considered to be differentially expressed with the threshold value setting to $|\log \mathrm{FC}|>2$ and $\mathrm{p}<0.05$.

To determine the specificity of DEGs between BeasSiNPs-P40 and Beas-P40 groups, bidirectional hierarchical clustering analysis with Euclidean distance (Szekely \& Rizzo 2005) was performed for the top 50 DEGs using pheatmap package in R (v1.0.8; http://cran.rproject.org/web/packages/pheatmap/index.html).

\section{PPI network construction and module analysis}

The DEGs were imported into STRING database (v10.0; Search Tool for the Retrieval of Interacting Genes; http://string db.org/) (Szklarczyk et al. 2015) to obtain the PPI data. The PPI network was constructed and visualized using Cytoscape software (v2.8; www.cytoscape.org/) (Kohl et al. 2011). The hub genes with more interactions with other partners (degree) were 
128

129

130

131

132

133

134

135

136

137

138

139

140

141

142

143

144

145

146

147

148

selected and plotted with ggplot2 in R package (v3.4.1; http://www.R-project.org/).

Furthermore, the Molecular Complex Detection (MCODE) plugin of Cytoscape software was also employed to identify functionally related and highly interconnected clusters from the PPI network with degree cutoff of 6 , node score cutoff of 0.2 , k-core of 5 and maximum depth of 100 (ftp://ftp.mshri.on.ca/pub/BIND/Tools/MCODE) (Bader \& Hogue 2003). Sub-modules were defined to be significant with MCODE score $\geqslant 4$ and nodes $\geqslant 6$.

\section{miRNA-target genes interaction prediction}

Because there were some pre-miRNAs included in the DEGs, the pre-miRNA-mRNA interaction was also investigated to explore the potential regulatory mechanisms of mRNAs. mRNA targets of differentially expressed miRNAs (DE-miRNAs) were predicted using the miRWalk database (v2.0; http://zmf.umm.uni-heidelberg.de/apps/zmf/mirwalk2/)(Dweep \& Gretz 2015), which were then overlapped with the DEGs to obtain DE-miRNA-DEGs interaction relationships. Then, the DE-miRNA-DEG interaction network was constructed and visualized using Cytoscape software (Kohl et al. 2011).

\section{Function enrichment analysis}

Gene ontology (GO) biological process terms, Kyoto encyclopedia of genes and genomes (KEGG), and BioCarta pathways were also enriched for all DEGs, DEGs in PPI and miRNADEG interaction networks by the Database for Annotation, Visualization and Integrated Discovery (DAVID) online tool (v6.8; http://david.abcc.ncifcrf.gov) (Dennis et al. 2003). Pvalue $<0.05$ was chosen as the threshold value for functional enrichment analyses.

\section{Results}


149

150

151

152

153

154

155

156

157

158

159

160

161

162

163

164

165

166

167

168

169

\section{Identification of DEGS}

After preprocessing and data normalization (gene expression in all samples are shown in Supplemental Information 2), DEGs between BeasSiNPs-P40 and Beas-P40 groups were identified by the LIMMA method. As a result, 323 genes were considered to be differentially expressed due to exceeding the difference threshold $(|\operatorname{logFC}|>2$ and $p<0.05)$, including 280 downregulated (containing 12 pre-miRNAs) and 43 upregulated genes (containing 29 premiRNAs) (Figure 1), which was fewer than the study. All the DEGs are listed in in Supplemental Information 3.

\section{Function enrichment analysis of DEGS}

The above differential genes were subjected to the online tool DAVID for function enrichment analysis. As a result, $49 \mathrm{GO}$ biological process terms were enriched for downregulated DEGs, including response to reactive oxygen species (ROS) (i.e. SOD1, superoxide dismutase 1). Only 2 GO biological process terms were enriched for upregulated DEGs, mainly involving in protein translation (i.e. EIF4G2, eukaryotic translation initiation factor 4 gamma 2; PABPC1, poly(A) binding protein cytoplasmic 1) (Table 1).

KEGG pathway analyses were also performed by DAVID software. In line with the GO terms, the downregulated DEGs were predicted to participate in oxidative stress related metabolism pathways, such as Oxidative phosphorylation (i.e. ATP5H, ATP synthase, $\mathrm{H}+$ transporting, mitochondrial F0 complex, subunit D), while upregulated DEGs were enriched for Regulation of eIF4e and p70 S6 Kinase (i.e. EIF4G2, PABPC1) (Table 2). 
171 (Figure 2A), including 92 nodes (86 downregulated and 6 upregulated) and 291 edges

172 (interaction relationships, such as EIF4G2-PABPC1, SOD1-ATP5H) (Supplemental Information

4). After calculating the degree, PABPC1 (degree $=15)$, ATP5H (degree $=11$ ) and SOD1

$($ degree $=8)$ were suggested to be hub genes (Figure 2B).

Following cluster analysis using the MCODE algorithm, three significant modules were obtained (Figure 3). Function enrichment analysis showed that ATP5H was included in module 3 and participated in Oxidative phosphorylation pathway (Table 3).

miRNA-mRNA interaction network

A total of 18896 target genes were predicted for the 41 DE-miRNAs by using the miRWalk database, 130 of which (including 124 downregulated and 6 upregulated) were found to be shared with the DEGs. Then, a miRNA-mRNA interaction network was constructed by using the

130 target genes and their corresponding 24 miRNAs, resulting in 1615 and 17 interrelationship

pairs for the 18 upregulated miRNAs and 6 downregulated miRNAs, respectively (Figure 4).

Function enrichment analysis also demonstrated these 130 target genes were involved in

GO terms of response to reactive oxygen species (hsa-miR-

3155b/3648/4522/4523/4533/4634/4734/572/661/933/1470-SOD1), and translational initiation

(miR-5095/548ai/622/4521-EIF4G2, miR-4521-PABPC1) (Table 4) and KEGG pathways of

Oxidative

phosphorylation

(hsa-miR-

1976/3155/3648/4461/4522/4523/4533/4634/4734/572/661/933-ATP5H) (Table 5).

\section{Discussion}


After PPI hub genes and module analysis as well as miRNA target gene prediction, our

192

193

194

195

196

197

198

199

200

201

202

203

204

205

206

207

208

209

210

211

present study preliminarily suggests downregulated ATP5H, SOD1 and upregulated EIF4G2,

PABPC1 may be especially important genes involved in amorphous SiNPs-mediated tumor initiation. EIF4G2 and PABPC1 may be involved in the progression of cancer by affecting translational initiation, while SOD1 and ATP5H may participate in carcinogenesis via influencing oxidative stress and Oxidative phosphorylation. Although genes were not similar, these enriched pathways seemed to be in line with the study of Guo et al. (Guo et al. 2017), further indicating these biological processes may be crucial.

Recently, accumulating evidence has indicated exposure to amorphous SiNPs induces oxidative stress in cells or organ (Guo et al.2015; Wu et al. 2016; Nemmar et al. 2016). For example, Jiang et al. incubated the erythrocytes with SiNPs and found the oxidative damage biomarker malondialdehyde (MDA) was significantly increased, while the activity of antioxidant superoxide dismutase (SOD) was decreased (Jiang et al. 2016). Di et al. showed exposure of SiNPs to macrophages elicited a greater oxidative stress that was assessed from heme oxygenase-1 (Hmox1) induction and ROS production (Di et al. 2016). Nemmar et al. observed intraperitoneal administration of SiNPs induced significantly increased lipid peroxidation in the lung, liver, kidney, and brain of mice, displaying reduced SOD and catalase activities (Nemmar et al. 2016). Consistent with these studies, our study also found anti-oxidative gene SOD1 was significantly downregulated after SiNPs treatment. The increased oxidative stress was reported to induce the switch of glucose metabolism from oxidative phosphorylation to aerobic glycolysis (the Warburg Effect) to promote excessive proliferation and growth of cells, leading to the 
212 development and progression of cancer (Pani et al. 2010; Molavian et al. 2016). ATP synthase is

213 an enzyme to be responsible for the synthesis of abundant ATP in oxidative phosphorylation

214 process. The expression of ATP synthase may be reduced due to the decreased oxidative

215 phosphorylation. As expected, Feichtinger et al. found significantly reduced levels of ATP

216 Synthase Subunit ATP5F1A was correlated with earlier-onset prostate cancer (Feichtinger et al.

217 2018). Shin et al. used the two-dimensional gel electrophoresis data to demonstrate the

218 expression and activity of ATP synthase were lower in 5-FU-resistant cells compared with parent

219 cancer cells. Inhibition of ATP synthase by oligomycin A or siRNA transfection strongly

220 antagonized 5-FU-induced suppression of cell proliferation and increased cell viability (Shin et

221 al. 2005). Similarly, the study of Song et al. indicated loss of ATP5H conferred a stem-like,

222 invasive phenotype to tumor cells as well as multimodal resistance to immunotherapy,

223 chemotherapy, and radiotherapy (Song et al. 2018). Also, reduced levels of ATP synthase were

224 associated with poor prognosis in cancer patients (Song et al. 2018). In agreement with these

225 studies, we also found ATP5H could interact with SOD1 and was downregulated in malignant

226 transformation after SiNPs treatment.

227 In accordance with our hypothesis that the increased oxidative stress and aerobic glycolysis

228 may accelerate the cell proliferation, we also found translational initiation was abnormally

229 activated in human lung epithelial cells after SiNPs exposure, which may, on one hand, promote

230 cell division and on the other hand, maintains cell survival (Pyronnet \& Sonenberg 2001).

231 Translation process requires the protein complex known as eukaryotic initiation factor 4F (eIF4F)

232 which consists of cap-binding protein eIF4E, scaffolding protein eIF4G, and ATP-dependent 
233 RNA helicase eIF4A (Gingras et al. 1999). Hereby, the expression of translation protein related

234 genes may be upregulated in malignant transformation, which have been confirmed in several

235 cancers because downregulation of eIF4GII was reported to decrease cell proliferation, but

236 induces cellular senescence (Emmrich et al. 2014; Xie et al. 2017). During translation process in

237 eukaryotic cells, nonsense mutations (premature stop codon) may be present, which disrupts

238 production of full-length, functional proteins (such as tumor suppressor gene p53) and thus may

239 induce the development of various diseases, including cancers (Kashofer et al. 2017). Nonsense-

240 mediated mRNA decay (NMD) represents a surveillance mechanism that eliminates transcripts

241 with nonsense mutations and prevents cancer development. In contrast, inhibition of NMD may

242 result in the initiation of cancer (Cao et al. 2017). Recent studies showed PABPC1 can interact

243 with eIF4G to inhibit NMD (Fatscher et al. 2014; Peixeiro et al. 2012). Thus, the upregulation of

244 PABPC1 may be one underlying reason for tumor formation. This hypothesis has been

245 demonstrated in the gastric carcinoma (Zhu et al. 2015) and hepatocellular carcinoma (Zhang et

246 al. 2015) samples. As expected, our present study also demonstrated SiNPs may induce the

247 tumorigenesis of Beas-2B cells by upregulating EIF4G2 and PABPC1. These two genes could

248 interact with each other.

249 More interestingly, our study showed the downregulation of ATP5H and SOD1 may be

250 resulted from the upregulation of their common upstream miR-3648/572/661, while the

251 downregulated miR-4521 may lead to the upregulation of the transcription of EIF4G2 and

252 PABPC1. Although all these were the potential mechanisms firstly obtained for the

253 carcinogenicity of amorphous SiNPs, recent studies on the miR-3648/572/661 and miR-4521 
254 may indirectly demonstrate their importance in cancer development. For example, Rashid et al.

255 demonstrated overexpression of miR-3648 promoted the growth of Hela cells, while opposite

256 results were obtained when miR-3648 was inhibited by antagomir. The mechanism of miR-3648

257 for cancer progression was to suppress the expression of a tumor suppressor gene adenomatous

258 polyposis coli 2 (APC2) (Rashid et al. 2017). miR-572 was also found to be highly expressed in

259 human ovarian cancer tissues and cell lines. Ectopic overexpression of miR-572 promoted

260 ovarian cancer cell proliferation and cell cycle progression in vitro and tumorigenicity in vivo by

261 inhibiting its direct targets suppressor of cytokine signaling 1 (SOCS1), cyclin-dependent kinase

262 inhibitor 1A (p21KIP). Kaplan-Meir analysis indicated that high level expression of miR-572

263 was associated with poorer overall survival (Zhang et al. 2015). miR-572 also can induce

264 proliferation, invasion and inhibit apoptosis of nasopharyngeal carcinoma cells by targeting

265 protein phosphatase 2 regulatory subunit Bgamma (PPP2R2C) (Yan et al. 2017). miR-661 was

266 observed to be upregulated in non-small cell lung cancer (NSCLC) tissues as compared to paired

267 adjacent tissues. Furthermore, miR-661 promoted proliferation, migration and metastasis of

268 NSCLC by regulating RB1 and mediating epithelial-mesenchymal transition process in NSCLC

269 (Liu et al. 2017). Yamaguchi et al. observed miR-4521 was downregulated in chemotherapy-

270 resistant renal cell carcinoma (Yamaguchi et al. 2017). However, the studies on miR-

$2713648 / 572 / 661$ and miR-4521 remains rare and their regulatory relationship with our predicted

272 target genes have not been investigated.

273 However, there are some limitations in this study. First, this is a preliminary study to 274 identify the potential carcinogenic mechanisms of amorphous SiNPs. Additional wet 
275

276

277

experiments are necessary to confirm the expressions of identified genes and miRNAs (i.e. PCR), their interaction relationships (i.e. dual-luciferase, overexpression or knockout in vitro and in vivo) as well as the influence on the cell proliferation, apoptosis, migration, and invasion. Second, our obtained miRNAs from the transcriptome array may be pre-miRNAs. Thus, further miRNA microarray (such as Affymetrix GeneChip miRNA v4) or sequencing analysis is essential to screen more crucial mature miRNAs.

\section{Conclusion}

Our findings reveal amorphous SiNPs may exert a carcinogenic effect by targeted regulating miR-3648/572/661 and miR-4521 followed by influencing their downstream target genes (ATP5H/SOD1 and EIF4G2/PABPC1), respectively. These target genes may be involved in cancer development by promoting the oxidative stress, translational initiation, but inhibiting Oxidative phosphorylation and NMD. Accordingly, the four miRNAs and their target genes may be underlying biomarkers for prediction of carcinogenesis when exposure to SiNPs and potential targets for cancer treatment.

\section{Acknowledgements}

None.

\section{Data Availability Statement}

Raw data is available in the Supplemental Materials (Supplemental Information 1).

\section{References}

Bader GD, and Hogue CW. 2003. An automated method for finding molecular complexes in large protein interaction networks. BMC Bioinformatics 4:2. 
296

297

298

299

300

301

302

303

304

305

306

307

308

309

310

311

312

313

314

315

316

Cao L, Qi L, Zhang L, Song W, Yu Y, Xu C, Li L, Guo Y, Yang L, Liu C, Huang Q, Wang Y, Sun B, Meng B, Zhang B, Cao W. 2017. Human nonsense-mediated RNA decay regulates EMT by targeting the TGF- $\beta$ signaling pathway in lung adenocarcinoma. Cancer Lett 403:246-259.

Dennis G Jr, Sherman BT, Hosack DA, Yang J, Gao W, Lane HC, Lempicki RA. 2003. DAVID: Database for Annotation, Visualization, and Integrated Discovery. Genome Biol 4:P3.

Di CL, Movia D, Bianchi MG, Allegri M, Mohamed BM, Bell AP, Moore C, Pinelli S, Rasmussen K, and Riegosintes J. 2016. Pro-inflammatory effects of pyrogenic and precipitated amorphous silica nanoparticles in innate immunity cells. Toxicol Sci 150:4053.

Dweep H, and Gretz N. 2015. miRWalk2.0: a comprehensive atlas of microRNA-target interactions. Nat Methods 12:697.

Emmrich S, Engeland F, El-Khatib M, Henke K, Obulkasim A, Schöning J, Katsman-Kuipers JE, Michel Zwaan C, Pich A, Stary J, Baruchel A, de Haas V, Reinhardt D, Fornerod M, van den Heuvel-Eibrink MM, Klusmann JH. 2016. miR-139-5p controls translation in myeloid leukemia through EIF4G2. Oncogene 35:1822-1831.

Fatscher T, Boehm V, Weiche B, Gehring NH. 2014. The interaction of cytoplasmic poly(A)binding protein with eukaryotic initiation factor 4G suppresses nonsense-mediated mRNA decay. RNA 20:1579-1592.

Feichtinger RG, Schäfer G, Seifarth C, Mayr JA, Kofler B, Klocker H. 2018. Reduced Levels of ATP Synthase Subunit ATP5F1A Correlate with Earlier-Onset Prostate Cancer. Oxid 
318 Fontana C, Kirsch A, Seidel C, Marpeaux L, Darne C, Gaté L, Remy A, and Guichard Y. 2017. In vitro cell transformation induced by synthetic amorphous silica nanoparticles. Mutat Res 823:22-27.

321

322

323

324

325

326

327

328

329

330

331

332

333

334

335

336

Fruijtier-Pölloth C. 2012. The toxicological mode of action and the safety of synthetic amorphous silica-a nanostructured material. Toxicology 294:61-79.

Gingras AC, Raught B, Sonenberg N. 1999 eIF4 initiation factors: effectors of mRNA recruitment to ribosomes and regulators of translation. Annu Rev Biochem 68: 913-963.

Guo C, Xia Y, Niu P, Jiang L, Duan J, Yu Y, Zhou X, Li Y, Sun Z. 2015. Silica nanoparticles induce oxidative stress, inflammation, and endothelial dysfunction in vitro via activation of the MAPK/Nrf2 pathway and nuclear factor- $\mathrm{BB}$ signaling. Int $J$ Nanomedicine 10:1463-1477.

Guo C, Wang J, Yang M, Li Y, Cui S, Zhou X, Li Y, and Sun Z. 2017. Amorphous silica nanoparticles induce malignant transformation and tumorigenesis of human lung epithelial cells via P53 signaling. Nanotoxicology 11:1176-1194.

Irizarry RA, Bolstad BM, Collin F, Cope LM, Hobbs B, and Speed TP. 2003. Summaries of Affymetrix GeneChip probe level data. Nucleic Acids Res 31:e15.

Jiang L, Yu Y, Li Y, Yu Y, Duan J, Zou Y, Li Q, and Sun Z. 2016. Oxidative Damage and Energy Metabolism Disorder Contribute to the Hemolytic Effect of Amorphous Silica Nanoparticles. Nanoscale Res Lett 11:57. 
338

339

340

341

342

343

344

345

346

347

348

349

350

351

352

353

354

355

356

357

358

661 promotes tumor invasion and metastasis by directly inhibiting RB1 in non small cell lung cancer. Mol Cancer 16:122.

Kashofer K, Winter E, Halbwedl I, Thueringer A, Kreiner M, Sauer S, Regauer S. 2017. HPVnegative penile squamous cell carcinoma: disruptive mutations in the TP53 gene are common. Mod Pathol 30:1013-1020.

Kohl M, Wiese S, and Warscheid B. 2011. Cytoscape: software for visualization and analysis of biological networks. Methods Mol Biol 696:291-303.

Liang XJ, Chen C, Zhao Y, Jia L, and Wang PC. 2008. Biopharmaceutics and therapeutic potential of engineered nanomaterials. Current Drug Metabolism 9:697-709.

Molavian HR, Kohandel M, Sivaloganathan S. 2016. High Concentrations of $\mathrm{H}_{2} \mathrm{O}_{2}$ Make Aerobic Glycolysis Energetically More Favorable for Cellular Respiration. Front Physiol $7: 362$.

Nemmar A, Yuvaraju P, Beegam S, Yasin J, Kazzam EE, and Ali BH. 2016. Oxidative stress, inflammation, and DNA damage in multiple organs of mice acutely exposed to amorphous silica nanoparticles. Int J Nanomedicine 11:919-928.

Pani G, Galeotti T, Chiarugi P. 2010 Metastasis: cancer cell's escape from oxidative stress. Cancer Metastasis 29:351-378.

Peixeiro I, Inácio Â, Barbosa C, Silva AL, Liebhaber SA, Romão L. 2012. Interaction of PABPC1 with the translation initiation complex is critical to the NMD resistance of AUG-proximal nonsense mutations. Nucleic Acids Res 40:1160-1173.

Peng F, Su Y, Zhong Y, Fan C, Lee ST, and He Y. 2014. Silicon Nanomaterials Platform for 
359

360

361

362

363

364

365

366

367

368

369

370

371

372

373

374

375

376

377

378

379

Bioimaging, Biosensing, and Cancer Therapy. Acc Chem Res 47:612-623.

Pyronnet S, and Sonenberg N. 2001. Cell-cycle-dependent translational control. Curr Opin Genet Dev 11:13-18.

Rashid F, Awan HM, Shah A, Chen L, Shan G. 2017. Induction of miR-3648 Upon ER Stress and Its Regulatory Role in Cell Proliferation. Int J Mol Sci 18: pii: E1375.

Ritchie ME, Phipson B, Wu D, Hu Y, Law CW, Shi W, and Smyth GK. 2015. limma powers differential expression analyses for RNA-sequencing and microarray studies. Nucleic Acids Res 43:e47.

Shin YK, Yoo BC, Chang HJ, Jeon E, Hong SH, Jung MS, Lim SJ, Park JG. 2005. Downregulation of mitochondrial F1F0-ATP synthase in human colon cancer cells with induced 5-fluorouracil resistance. Cancer Res 65:3162-3170.

Song KH, Kim JH, Lee YH, Bae HC, Lee HJ, Woo SR, Oh SJ, Lee KM, Yee C, Kim BW, Cho H, Chung EJ, Chung JY, Hewitt SM, Chung TW, Ha KT, Bae YK, Mao CP, Yang A, Wu TC, Kim TW. 2018. Mitochondrial reprogramming via ATP5H loss promotes multimodal cancer therapy resistance. J Clin Invest 128:4098-4114.

Szekely GJ, and Rizzo ML. 2005. Hierarchical Clustering via Joint Between-Within Distances: Extending Ward's Minimum Variance Method. J Classif 22:151-183.

Szklarczyk D, Franceschini A, Wyder S, Forslund K, Heller D, Huerta-Cepas J, Simonovic M, Roth A, Santos A, and Tsafou KP. 2015. STRING v10: protein-protein interaction networks, integrated over the tree of life. Nucleic Acids Res 43:D447.

Wu J, Shi Y, Asweto CO, Feng L, Yang X, Zhang Y, Hu H, Duan J, Sun Z. 2016. Co-exposure 
380

381

382

383

384

385

386

387

388

389

390

391

392

393

394

395

396

397

398

399

400 to amorphous silica nanoparticles and benzo[a]pyrene at low level in human bronchial epithelial BEAS-2B cells. Environ Sci Pollut Res Int 23:23134-23144.

Xie X, Li YS, Xiao WF, Deng ZH, He HB, Liu Q, Luo W. 2017. MicroRNA-379 inhibits the proliferation, migration and invasion of human osteosarcoma cells by targetting EIF4G2. Biosci Rep 37: pii: BSR20160542.

Yamaguchi N, Osaki M, Onuma K, Yumioka T, Iwamoto H, Sejima T, Kugoh H, Takenaka A, Okada F. 2017. Identification of MicroRNAs Involved in Resistance to Sunitinib in Renal Cell Carcinoma Cells. Anticancer Res 37:2985-2992.

Yan L, Cai K, Liang J, Liu H, Liu Y, Gui J. 2017. Interaction between miR-572 and PPP2R2C, and their effects on the proliferation, migration, and invasion of nasopharyngeal carcinoma (NPC) cells. Biochem Cell Biol 95:578-584.

Zhang H, Sheng C, Yin Y, Wen S, Yang G, Cheng Z, Zhu Q. 2015. PABPC1 interacts with AGO2 and is responsible for the microRNA mediated gene silencing in high grade hepatocellular carcinoma. Cancer Lett 367:49-57.

Zhang X, Liu J, Zang D, Wu S, Liu A, Zhu J, Wu G, Li J, Jiang L. 2015. Upregulation of miR572 transcriptionally suppresses SOCS1 and p21 and contributes to human ovarian cancer progression. Oncotarget 6:15180-15193.

Zhu J, Ding H, Wang X, Lu Q. 2015. PABPC1 exerts carcinogenesis in gastric carcinoma by targeting miR-34c. Int J Clin Exp Pathol 8:3794-3802. 
401

402

403

404

405

406

407

408

409

410

411

412

413

414

415

416

417

418

419 Figure legends:

420 Figure 1: Heat map of differentially expressed genes identified between human lung epithelial 421 cell Beas-2B exposed to amorphous silica nanoparticles or not. High level of expression was 
422 indicated as red and low level was in green. Heatmap was established based on normalized

423 expression values of significantly changed mRNAs. The expression values are depicted in line

424 with the color scale. The intensity increases from green to red. Each column represents one 425 sample, and each row indicates a transcript.

426 Figure 2: Protein-protein interaction network to screen crucial genes. A, protein-protein 427 interaction network of differentially expressed genes between human lung epithelial cell Beas428 2B exposed to amorphous silica nanoparticles or not. Downregulated genes were indicated as 429 orange and upregulated genes were in green. B, proteins rank according their interaction pairs in 430 the protein-protein interaction network.

431 Figure 3: Modules obtained from PPI network. Orange, downregulated genes; green, 432 upregulated genes. A, module 1; B, module 2; C, module 3.

433 Figure 4: A miRNA-gene interaction network. Blue diamond, upregulated miRNAs; yellow 434 diamond, downregulated miRNAs; red oval, downregulated differentially expressed genes; green, 435 upregulated differentially expressed genes.

436 Supplementary Materials:

437 Supplemental Information 1: Raw data used in this manuscript. 1.1: GSM2182800; 1.2: 438 GSM2182801; 1.3: GSM2182802; 1.4: GSM2182803; 1.5: GSM2182804; 1.6: GSM2182805.

439 Supplemental Information 2: Processed genes in all samples.

440 Supplemental Information 3: All differentially expressed genes.

441 Supplemental Information 4: Protein-protein interaction data. 


\section{Figure 1}

Heat map of differentiallyexpressed genes identified between human lung epithelial cell Beas-2B exposedto amorphous silica nanoparticles or not.

High level of expression was indicated as red and low level was in green. Heatmap was established based on normalized expression values of significantly changed mRNAs. The expression values are depicted in line with the color scale. The intensity increases from green to red. Each column represents one sample, and each row indicates a transcript. 


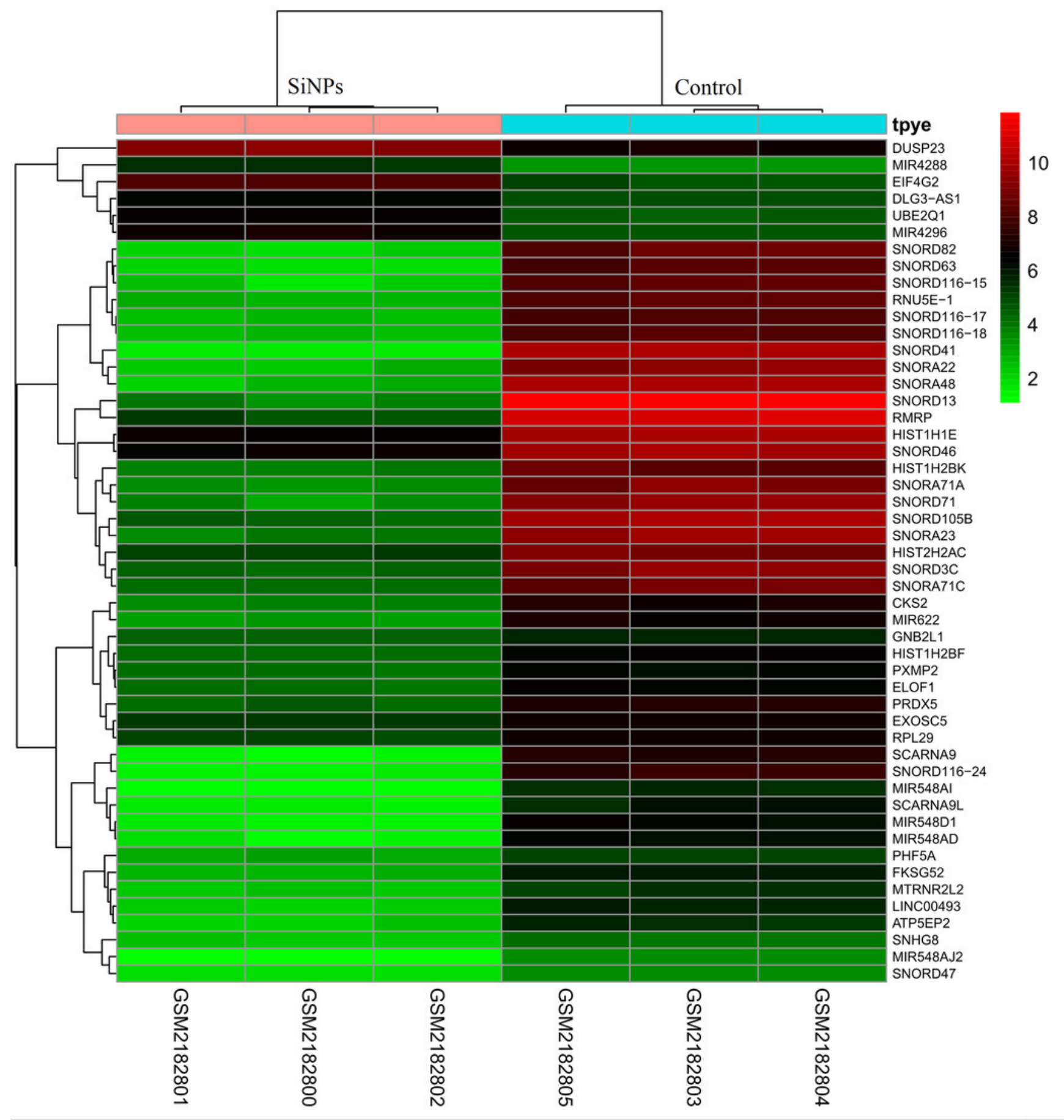


Figure 2

Protein-protein interaction network to screen crucial genes

A, protein-protein interaction network of differentially expressed genes between human lung epithelial cell Beas-2B exposed to amorphous silica nanoparticles or not. Downregulated genes were indicated as orange and upregulated genes were in green. B, proteins rank according their interaction pairs in the protein-protein interaction network.
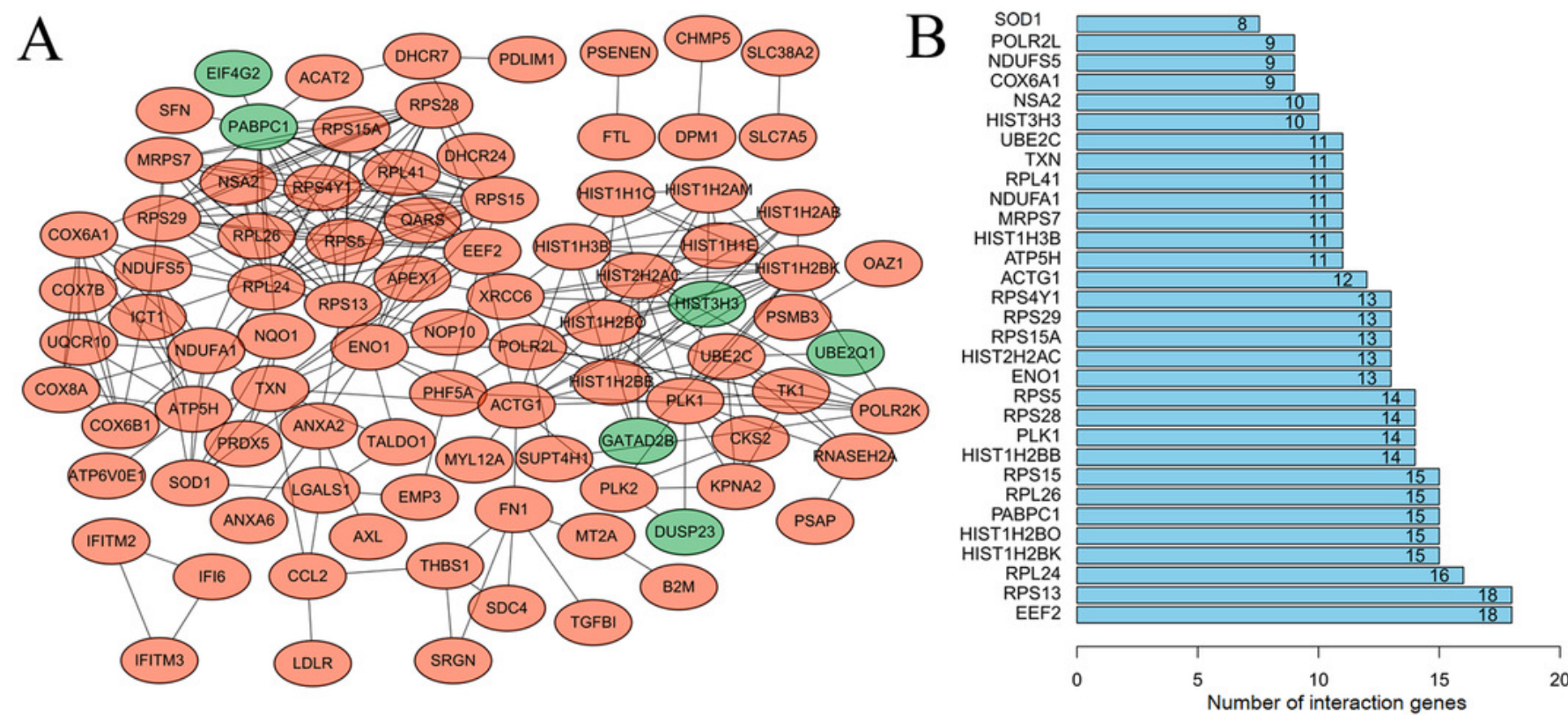
Figure 3

Modules obtained from PPI network.

Orange, upregulated genes; green, down-regulated genes. A, module 1; B, module 2; C, module 3.

A

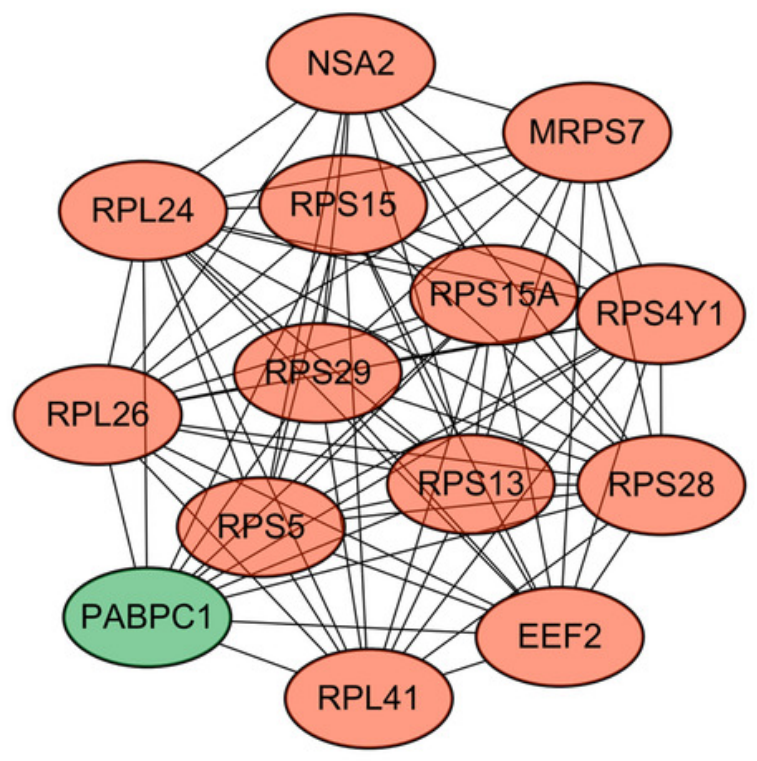

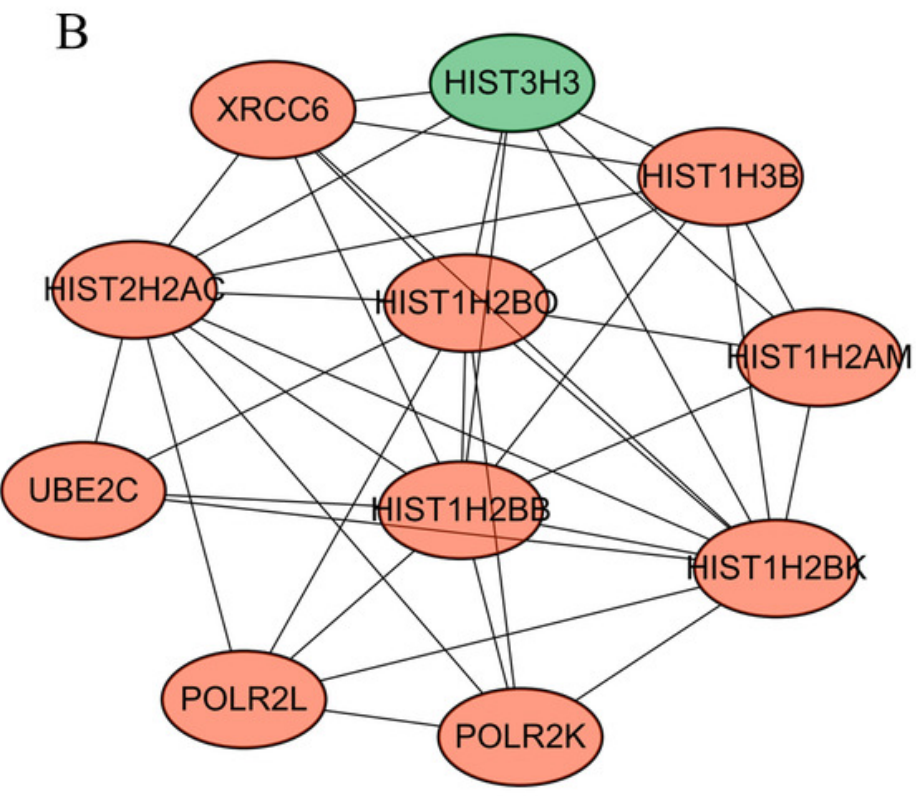

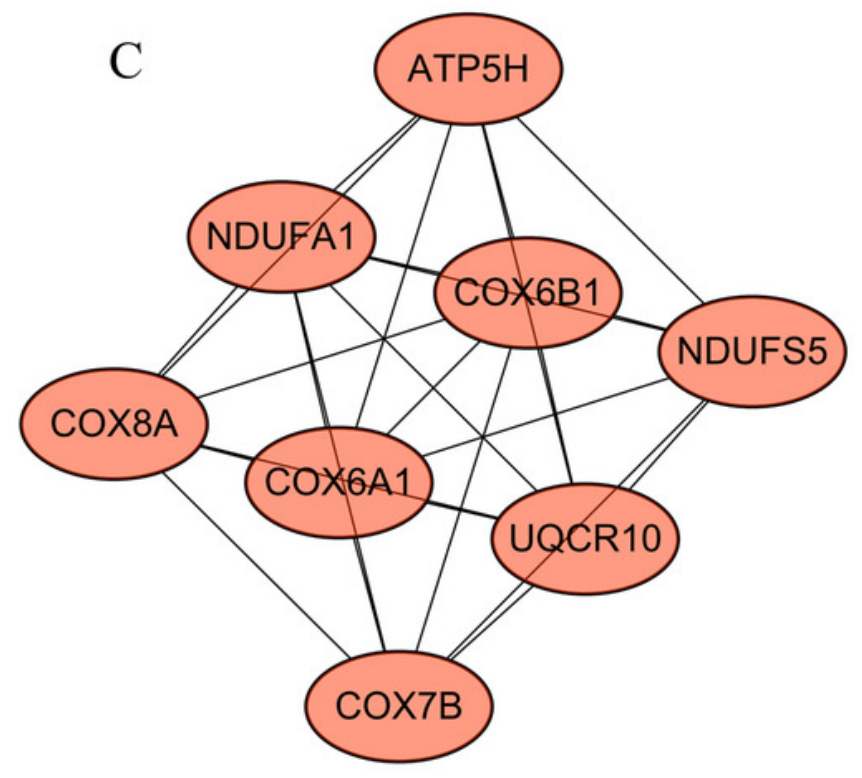




\section{Figure 4}

A miRNA-gene interaction network.

Orange, up-regulated genes; green, down-regulated genes. Blue diamond, downregulated miRNAs; yellow diamond, upregulated miRNAs; red oval, upregulated differentially expressed genes; green, downregulated differentially expressed genes.
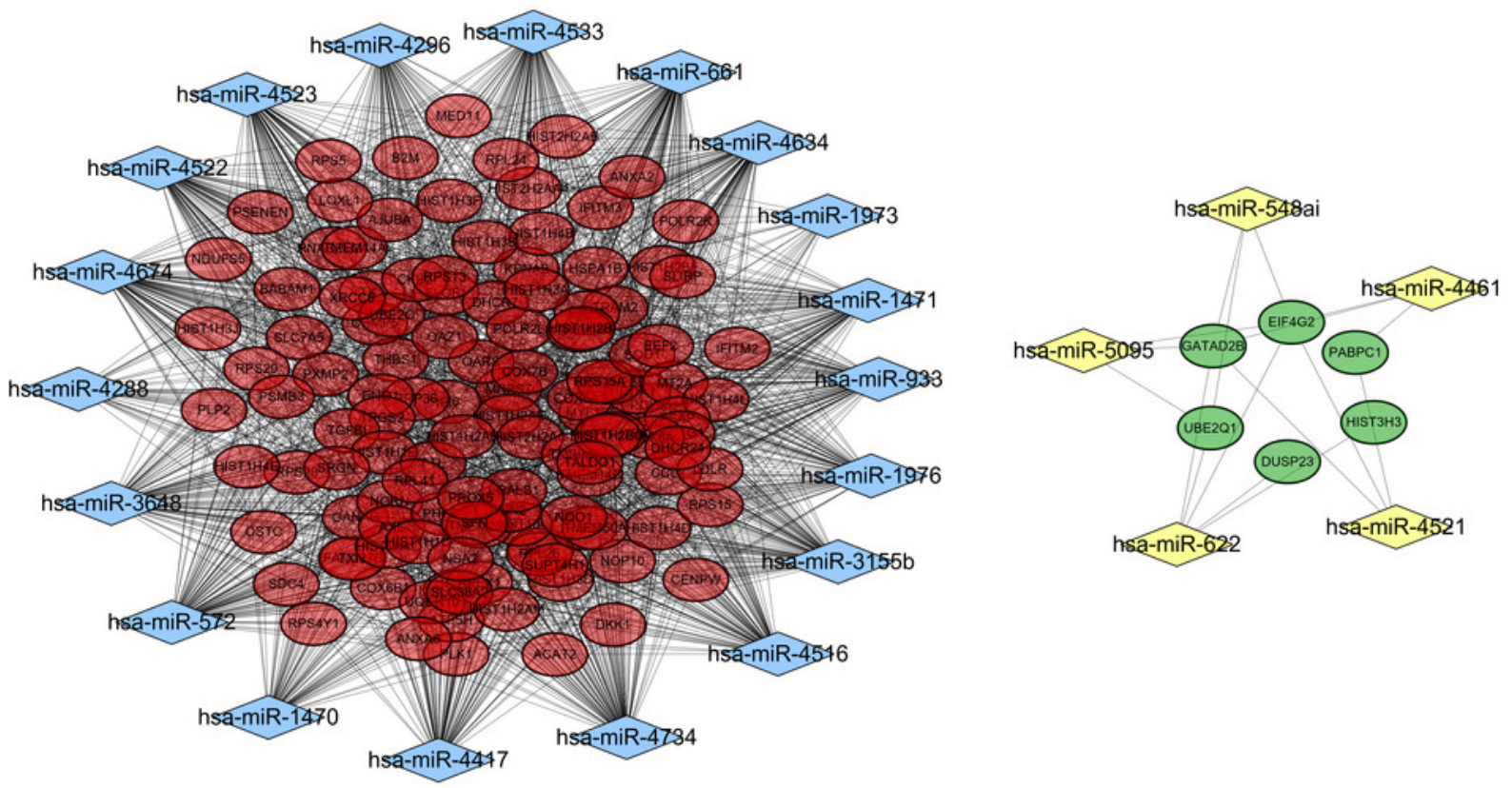


\section{Table $\mathbf{1}$ (on next page)}

Gene ontology (GO) biological process terms analysis for all differentially expressed genes 
1 Table 1 Gene ontology (GO) biological process terms analysis for all differentially expressed genes

\begin{tabular}{ll}
\hline & Term \\
\hline Down & GO:0006334 nucleosome assembly
\end{tabular}

GO:0051290 protein

heterotetramerization

GO:0032200 telomere organization

GO:0045815 positive regulation of gene expression, epigenetic

GO:0006335 DNA

replication-

dependent nucleosome assembly

GO:0000183 chromatin silencing at rDNA

GO:0045814 negative regulation of gene expression, epigenetic

GO:003 1047 gene silencing by RNA

GO:0044267 cellular protein metabolic process

GO:0006614 SRP-dependent cotranslational protein targeting to membrane

GO:0098609 cell-cell adhesion

GO:0019083 viral transcription

GO:0000184 nuclear-transcribed mRNA catabolic process, nonsense-mediated

P-value Genes

2.32E-16 HIST1H2BB, HIST1H3J, HIST1H4L, HIST1H1E, HIST1H1C, HIST1H2BF, HIST1H2BO, HIST1H2BK, HIST1H4B, HIST1H2BI, HIST1H3A, HIST1H4E, HIST1H3B, HIST1H4C, HIST1H3D, HIST1H4D, HIST1H3F

5.54E-15 HIST1H3J, HIST1H4L, HIST1H4B, XRCC6, HIST1H3A, HIST1H3B, HIST1H4E, HIST1H3D, HIST1H4C, HIST1H4D, HIST1H3F, ANXA2

1.51E-13 HIST1H3J, HIST1H4L, HIST1H4B, HIST1H3A, HIST1H3B, HIST1H4E, HIST1H3D, HIST1H4C, HIST1H4D, HIST1H3F

5.82E-13 HIST1H3J, HIST1H4L, POLR2L, POLR2K, HIST1H4B, HIST1H3A, HIST1H3B, HIST1H4E, HIST1H3D, HIST1H4C, HIST1H4D, HIST1H3F

8.76E-13 HIST1H3J, HIST1H4L, HIST1H4B, HIST1H3A, HIST1H3B, HIST1H4E, HIST1H3D, HIST1H4C, HIST1H4D, HIST1H3F

3.77E-12 HIST1H3J, HIST1H4L, HIST1H4B, HIST1H3A, HIST1H3B, HIST1H4E, HIST1H3D, HIST1H4C, HIST1H4D, HIST1H3F

7.03E-11 HIST1H3J, HIST1H4L, HIST1H4B, HIST1H3A, HIST1H3B, HIST1H4E, HIST1H3D, HIST1H4C, HIST1H4D, HIST1H3F

4.04E-10 HIST1H3J, HIST1H4L, POLR2L, POLR2K, HIST1H4B, HIST1H3A, HIST1H3B, HIST1H4E, HIST1H3D, HIST1H4C, HIST1H4D, HIST1H3F

7.84E-10 HIST1H3J, HIST1H4L, HIST1H4B, TGFBI, HIST1H3A, HIST1H3B, HIST1H4E, HIST1H3D, HIST1H4C, HIST1H4D, HIST1H3F, B2M

2.29E-08 RPS28, RPS29, RPL41, RPS15, RPL26, RPS13, RPS15A, RPS4Y1, RPL24, RPS5

7.12E-08 HIST1H3J, CHMP5, EEF2, RPL24, PDLIM1, SFN, ANXA2, KRT18, HIST1H3A, HIST1H3B, HIST1H3D, CNN2, HIST1H3F, ENO1

1.06E-07 RPS28, RPS29, RPL41, RPS15, RPL26, RPS13, RPS15A, RPS4Y1, RPL24, RPS5

1.80E-07 RPS28, RPS29, RPL41, RPS15, RPL26, RPS13, RPS15A, RPS4Y1, RPL24, RPS5 
decay

GO:0006413 translational initiation

GO:0060968 regulation of gene silencing

GO:0006364 rRNA processing

GO:0006303 double-strand break repair via nonhomologous end joining

GO:0045653 negative regulation of 7.02E-06 megakaryocyte differentiation

GO:0034080 CENP-A containing

nucleosome assembly

GO:0006412 translation

GO:0006342 chromatin silencing

GO:0016233 telomere capping

GO:0006336 DNA replication-

independent nucleosome assembly

GO:0006352 DNA-templated

transcription, initiation

GO:1904837 beta-catenin-TCF complex assembly

GO:0000028 ribosomal small subunit assembly

GO:0006123 mitochondrial electron transport, cytochrome $\mathrm{c}$ to oxygen

GO:1902600 hydrogen

ion $\quad 9.45 \mathrm{E}-04$

transmembrane transport

GO:0043154 negative regulation of cysteine-type endopeptidase activity involved in apoptotic process

GO:0019731 antibacterial humoral $3.81 \mathrm{E}-03$ response

GO:0042274 ribosomal small subunit 5.69E-03 biogenesis

GO:0002576 platelet degranulation

GO:0002227 innate immune response in

6.37E-03

$1.36 \mathrm{E}-02$

$1.67 \mathrm{E}-05$
RPS28, RPS29, RPL41, RPS15, RPL26, RPS13, RPS15A, RPS4Y1, RPL24, RPS5

HIST1H3J, HIST1H3A, HIST1H3B, HIST1H3D, HIST1H3F

RPS28, RPS29, RPL41, RRP36, RPS15, RPL26, RPS13, RPS15A, RPS4Y1, RPL24, RPS5

HIST1H4L, HIST1H4B, XRCC6, HIST1H4E, BABAM1, HIST1H4C, HIST1H4D

HIST1H4L, HIST1H4B, HIST1H4E, HIST1H4C, HIST1H4D

HIST1H4L, HIST1H4B, HIST1H4E, CENPW, HIST1H4C, HIST1H4D

1.37E-05 RPS28, RPS29, RPL41, RPS15, RPL26, RPS13, RPS15A, RPS4Y1, RPL24, MRPS7, RPS5

HIST1H2AB, HIST2H2AB, HIST2H2AA4, HIST2H2AC, HIST1H2AE, HIST1H2AM

1.98E-05 HIST1H4L, HIST1H4B, HIST1H4E, HIST1H4C, HIST1H4D

HIST1H4L, HIST1H4B, HIST1H4E, HIST1H4C, HIST1H4D

HIST1H4L, HIST1H4B, HIST1H4E, HIST1H4C, HIST1H4D

HIST1H4L, HIST1H4B, HIST1H4E, HIST1H4C, HIST1H4D

RPS28, RPS15, MRPS7, RPS5

COX7B, COX8A, COX6B1, COX6A1

UQCR10, COX7B, COX8A, COX6B1, COX6A1

PRDX5, SFN, THBS1, IFI6, DHCR24

HIST1H2BK, HIST1H2BF, HIST1H2BI, B2M

RPS28, RRP36, RPS15

PSAP, THBS1, SOD1, SRGN, FN1

HIST1H2BK, HIST1H2BF, HIST1H2BI 
GO:0043086 negative regulation of 1.66E-02 OAZ1, NQO1, ANXA2, ANXA2P2

catalytic activity

GO:0050434 positive regulation of viral 1.81E-02 transcription

GO:0050830 defense response to Gram- $2.30 \mathrm{E}-02$ positive bacterium

GO:0006368 transcription elo
from RNA polymerase II promoter

GO:0033490 cholesterol

process via lathosterol

GO:0033489 cholesterol

biosynthetic $2.83 \mathrm{E}-02$

process via desmosterol

GO:0007568 aging

3.07E-02 CCL2, EEF2, SOD1, NQO1, APEX1

GO:0000302 response to

to reactive $3.16 \mathrm{E}-02$

oxygen species

GO:0001895 retina homeostasis

3.31E-02 ACTG1, SOD1, B2M

GO:0045471 response to ethanol

3.94E-02

GO:0006356 regulation of transcription

4.21E-02

from RNA polymerase I promoter

GO:0098532 histone

H3-K27 4.21E-02

trimethylation

GO:0007596 blood coagulation

4.30E-02

HIST1H3J, HIST1H3A, HIST1H3B, HIST1H3D, HIST1H3F

GO:0009615 response to virus

4.43E-02 IFITM2, IFITM3, RPS15A, ENO1

GO:0043066 negative regulation of apoptotic process

GO:1900121 negative regulation of receptor binding

Up
GO:0045727 positive regulation of translation

GO:0006413 translational initiation
1.57E-02 EIF4G2, PABPC1

4.01E-02 EIF4G2, PABPC1 


\section{Table 2 (on next page)}

KEGG pathway enrichment analysis for all differentially expressed genes 
1 Table 2 KEGG pathway enrichment analysis for all differentially expressed genes

\begin{tabular}{|c|c|c|c|}
\hline & Term & P-value & Genes \\
\hline \multirow[t]{16}{*}{ Down } & hsa05322:Systemic & $2.06 \mathrm{E}-16$ & HIST1H2AB, HIST1H2BB, HIST1H3J, HIST1H4L, \\
\hline & erythematosus & & HIST1H2BF, $\quad$ HIST1H2AE, \\
\hline & & & $\begin{array}{ll}\text { HIST1H2BO, HIST2H2AB, } & \text { HIST1H2BK, } \\
\text { HIST1H4B, HIST2H2AC, HIST1H2BI, HIST1H3A, } \\
\text { HIST1H4E, HIST1H3B, HIST1H3D, HIST1H4C, } \\
\text { HIST1H4D, HIST1H3F, HIST1H2AM }\end{array}$ \\
\hline & hsa05034:Alcoholism & $5.44 \mathrm{E}-14$ & HIST1H2AB, HIST1H2BB, HIST1H3J, HIST1H4L, \\
\hline & & & HIST2H2AA4, $\quad$ HIST1H2BF, $\quad$ HIST1H2AE, \\
\hline & & & HIST1H2BO, \\
\hline & & & $\begin{array}{l}\text { HIST1H4B, HIST2H2AC, HIST1H2BI, HIST1H3A, } \\
\text { HIST1H4E, HIST1H3B, HIST1H3D, HIST1H4C, } \\
\text { HIST1H4D, HIST1H3F, HIST1H2AM }\end{array}$ \\
\hline & hsa03010:Ribosome & $9.39 \mathrm{E}-06$ & $\begin{array}{l}\text { RPS28, RPS29, RPL41, RPS15, RPL26, RPS13, } \\
\text { RPS15A, RPS4Y1, RPL24, MRPS7, RPS5 }\end{array}$ \\
\hline & $\begin{array}{l}\text { hsa05016:Huntington's } \\
\text { disease }\end{array}$ & $1.80 \mathrm{E}-04$ & $\begin{array}{l}\text { UQCR10, NDUFS5, POLR2L, POLR2K, COX7B, } \\
\text { COX8A, COX6B1, COX6A1, SOD1, NDUFA1, } \\
\text { ATP5H }\end{array}$ \\
\hline & $\begin{array}{l}\text { hsa00190:Oxidative } \\
\text { phosphorylation }\end{array}$ & $3.14 \mathrm{E}-04$ & $\begin{array}{l}\text { UQCR10, NDUFS5, ATP6V0E1, COX7B, COX8A, } \\
\text { COX6B1, COX6A1, NDUFA1, ATP5H }\end{array}$ \\
\hline & $\begin{array}{l}\text { hsa05203:Viral } \\
\text { carcinogenesis }\end{array}$ & $1.32 \mathrm{E}-03$ & $\begin{array}{ll}\text { HIST1H2BO, } & \text { HIST1H2BB, } \\
\text { HIST1H2BK, HIST1H2BF, HIST1H4B, HIST1H2BI, } \\
\text { HIST1H4E, HIST1H4C, HIST1H4D }\end{array}$ \\
\hline & $\begin{array}{l}\text { hsa05010:Alzheimer's } \\
\text { disease }\end{array}$ & $1.47 \mathrm{E}-03$ & $\begin{array}{l}\text { UQCR10, NDUFS5, COX7B, COX8A, COX6B1, } \\
\text { COX6A1, PSENEN, NDUFA1, ATP5H }\end{array}$ \\
\hline & $\begin{array}{l}\text { hsa05012:Parkinson's } \\
\text { disease }\end{array}$ & $2.41 \mathrm{E}-03$ & $\begin{array}{l}\text { UQCR10, NDUFS5, COX7B, COX8A, COX6B1, } \\
\text { COX6A1, NDUFA1, ATP5H }\end{array}$ \\
\hline & $\begin{array}{l}\text { hsa04932:Non-alcoholic } \\
\text { fatty liver disease (NAFLD) }\end{array}$ & $1.36 \mathrm{E}-02$ & $\begin{array}{l}\text { UQCR10, NDUFS5, COX7B, COX8A, COX6B1, } \\
\text { COX6A1, NDUFA1 }\end{array}$ \\
\hline & $\begin{array}{l}\text { hsa04260:Cardiac muscle } \\
\text { contraction }\end{array}$ & $1.62 \mathrm{E}-02$ & UQCR10, COX7B, COX8A, COX6B1, COX6A1 \\
\hline & $\begin{array}{l}\text { hsa03008:Ribosome } \\
\text { biogenesis in eukaryotes }\end{array}$ & 2.64E-02 & $\begin{array}{l}\text { SNORD3A, SNORD3C, SNORD3B-1, SNORD3B-2, } \\
\text { NOP10 }\end{array}$ \\
\hline Up & $\begin{array}{l}\text { BIOCARTA } \\
\quad \text { h_eif4Pathway:Regula } \\
\text { tion of eIF4e and p70 S6 } \\
\text { Kinase }\end{array}$ & $1.48 \mathrm{E}-02$ & EIF4G2, PABPC1 \\
\hline
\end{tabular}

2 KEGG, Kyoto encyclopedia of genes and genomes. 


\section{Table 3(on next page)}

KEGG pathway enrichment analysis for DEGs in modules

DEGs, differentially expressed genes; KEGG, Kyoto encyclopedia of genes and genomes. 
1 Table 3 KEGG pathway enrichment analysis for DEGs in modules

\begin{tabular}{|c|c|c|c|}
\hline Module & Term & $\mathrm{P}$-value & Genes \\
\hline 1 & hsa03010:Ribosome & $8.25 \mathrm{E}-17$ & $\begin{array}{l}\text { RPS28, RPL41, RPS29, RPS15, } \\
\text { RPL26, RPS4Y1, RPS13, RPS15A, } \\
\text { RPL24, RPS5 }\end{array}$ \\
\hline 2 & $\begin{array}{l}\text { hsa05322:Systemic } \\
\text { lupus erythematosus }\end{array}$ & $9.23 \mathrm{E}-09$ & $\begin{array}{l}\text { HIST1H2BO, HIST1H2BB, } \\
\text { HIST1H2BK, HIST2H2AC, } \\
\text { HIST1H3B, HIST1H2AM, } \\
\text { HIST3H3 }\end{array}$ \\
\hline \multirow[t]{5}{*}{3} & $\begin{array}{l}\text { hsa05012:Parkinson's } \\
\text { disease }\end{array}$ & $5.44 \mathrm{E}-12$ & $\begin{array}{l}\text { NDUFS5, UQCR10, COX8A, } \\
\text { COX7B, COX6B1, COX6A1, } \\
\text { NDUFA1, ATP5H }\end{array}$ \\
\hline & $\begin{array}{l}\text { hsa00190:Oxidative } \\
\text { phosphorylation }\end{array}$ & $6.08 \mathrm{E}-12$ & $\begin{array}{l}\text { NDUFS5, UQCR10, COX8A, } \\
\text { COX7B, COX6B1, COX6A1, } \\
\text { NDUFA1, ATP5H }\end{array}$ \\
\hline & $\begin{array}{l}\text { hsa05010:Alzheimer's } \\
\text { disease }\end{array}$ & $3.06 \mathrm{E}-11$ & $\begin{array}{l}\text { NDUFS5, UQCR10, COX8A, } \\
\text { COX7B, COX6B1, COX6A1, } \\
\text { NDUFA1, ATP5H }\end{array}$ \\
\hline & $\begin{array}{l}\text { hsa05016:Huntington's } \\
\text { disease }\end{array}$ & $6.21 \mathrm{E}-11$ & $\begin{array}{l}\text { NDUFS5, UQCR10, COX8A, } \\
\text { COX7B, COX6B1, COX6A1, } \\
\text { NDUFA1, ATP5H }\end{array}$ \\
\hline & $\begin{array}{l}\text { hsa04260:Cardiac } \\
\text { muscle contraction }\end{array}$ & $1.73 \mathrm{E}-06$ & $\begin{array}{l}\text { UQCR10, COX8A, COX7B, } \\
\text { COX6B1, COX6A1 }\end{array}$ \\
\hline
\end{tabular}

2 DEGs, differentially expressed genes; KEGG, Kyoto encyclopedia of genes and genomes. 


\section{Table 4 (on next page)}

Gene ontology (GO) biological process terms analysis for DEGs in miRNA-mRNA regulatory network 
1 Table 4 Gene ontology (GO) biological process terms analysis for DEGs in miRNA-mRNA regulatory network

\begin{tabular}{|c|c|c|}
\hline Term & ralue & nes \\
\hline G & & 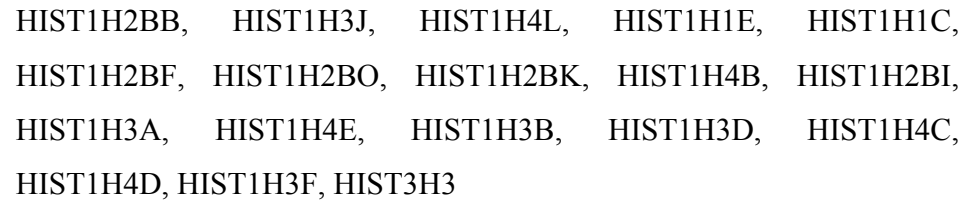 \\
\hline tetramerization & 16 & $\begin{array}{l}\text { HIST1H3J, HIST1H4L, XRCC6, ANXA2, HIST1H4B, HIST1H3A, } \\
\text { HIST1H4E, HIST1H3B, HIST1H4C, HIST1H3D, HIST1H4D, HIST3H3, } \\
\text { HIST1H3F }\end{array}$ \\
\hline GO:0032200 telomere organization & 13 & $\begin{array}{l}\text { HIST1H3J, HIST1H4L, HIST1H4B, HIST1H3A, HIST1H3B, HIST1H4E, } \\
\text { HIST1H3D, HIST1H4C, HIST1H4D, HIST1H3F }\end{array}$ \\
\hline $\begin{array}{l}\text { GO:0045815 positive regulation of gene } \\
\text { expression, epigenetic }\end{array}$ & $38 \mathrm{E}-13$ & $\begin{array}{l}\text { HIST1H3J, HIST1H4L, POLR2L, POLR2K, HIST1H4B, HIST1H3A, } \\
\text { HIST1H3B, HIST1H4E, HIST1H3D, HIST1H4C, HIST1H4D, HIST1H3F }\end{array}$ \\
\hline $\begin{array}{l}\text { GO:0006335 DNA } \\
\text { nucleosome assembly }\end{array}$ & $5 \mathrm{E}-13$ & $\begin{array}{l}\text { HIST1H3J, HIST1H4L, HIST1H4B, HIST1H3A, HIST1H3B, HIST1H4E, } \\
\text { HIST1H3D, HIST1H4C, HIST1H4D, HIST1H3F }\end{array}$ \\
\hline tin silencing at $\mathrm{rDNA}$ & -12 & $\begin{array}{l}\text { HIST1H3J, HIST1H4L, HIST1H4B, HIST1H3A, HIST1H3B, HIST1H4E, } \\
\text { HIST1H3D, HIST1H4C, HIST1H4D, HIST1H3F }\end{array}$ \\
\hline qene silencing by RNA & E-11 & $\begin{array}{l}\text { HIST1H3J, HIST1H4L, POLR2L, POLR2K, HIST1H4B, HIST1H3A, } \\
\text { HIST1H4E, HIST1H3B, HIST1H4C, HIST1H3D, HIST1H4D, PABPC1, } \\
\text { HIST1H3F }\end{array}$ \\
\hline $\begin{array}{l}\text { GO:0045814 negative regulation of gene } \\
\text { expression, epigenetic }\end{array}$ & & $\begin{array}{l}\text { HIST1H3J, HIST1H4L, HIST1H4B, HIST1H3A, HIST1H3B, HIST1H4E, } \\
\text { HIST1H3D, HIST1H4C, HIST1H4D, HIST1H3F }\end{array}$ \\
\hline $\begin{array}{l}\text { GO:0044267 cellular protein metabolic } \\
\text { process }\end{array}$ & -10 & $\begin{array}{l}\text { HIST1H3J, HIST1H4L, HIST1H4B, TGFBI, HIST1H3A, HIST1H3B, } \\
\text { HIST1H4E, HIST1H3D, HIST1H4C, HIST1H4D, HIST1H3F, B2M }\end{array}$ \\
\hline GO:0006413 translational initiation & -09 & $\begin{array}{l}\text { EIF4G2, RPS28, RPS29, RPL41, RPS15, RPL26, RPS13, RPS15A, } \\
\text { RPS4Y1, RPL24, PABPC1, RPS5 }\end{array}$ \\
\hline GO:0098 & & $\begin{array}{l}\text { HIST1H3J, CHMP5, PDLIM1, EEF2, RPL24, SFN, ANXA2, EIF4G2, } \\
\text { KRT18, HIST1H3A, HIST1H3B, HIST1H3D, CNN2, HIST1H3F, ENO1 }\end{array}$ \\
\hline $\begin{array}{l}\text { GO:0000184 nuclear-transcribed mRNA } \\
\text { catabolic process, nonsense-mediated decay }\end{array}$ & -08 & $\begin{array}{l}\text { RPS28, RPS29, RPL41, RPS15, RPL26, RPS13, RPS15A, RPS4Y1, } \\
\text { RPL24, PABPC1, RPS5 }\end{array}$ \\
\hline $\begin{array}{l}\text { GO:0006614 SRP-dependent cotranslational } \\
\text { protein targeting to membrane }\end{array}$ & $7 \mathrm{E}-08$ & $\begin{array}{l}\text { RPS28, RPS29, RPL41, RPS15, RPL26, RPS13, RPS15A, RPS4Y1, } \\
\text { RPL24, RPS5 }\end{array}$ \\
\hline GO:0019083 viral transcription & 4E-07 & $\begin{array}{l}\text { RPS28, RPS29, RPL41, RPS15, RPL26, RPS13, RPS15A, RPS4Y1, } \\
\text { RPL24, RPS5 }\end{array}$ \\
\hline $\begin{array}{l}\text { GO:0006303 double-strand break repair via } \\
\text { nonhomologous end joining }\end{array}$ & $3.36 \mathrm{E}-07$ & $\begin{array}{l}\text { HIST1H4L, HIST1H4B, XRCC6, HIST1H4E, BABAM1, HIST1H4C, } \\
\text { HIST1H4D, HIST3H3 }\end{array}$ \\
\hline GO:0016233 telomere capping & 42E-07 & HIST1H4L, HIST1H4B, HIST1H4E, HIST1H4C, HIST1H4D, HIST3H3 \\
\hline ion of gene silencing & E-07 & HIST1H3J, HIST1H3A, HIST1H3B, HIST1H3D, HIST1H3F \\
\hline GO:0006364 rRNA processing & $3.38 \mathrm{E}-06$ & $\begin{array}{l}\text { RPS28, RPS29, RPL41, RRP36, RPS15, RPL26, RPS13, RPS15A, } \\
\text { RPS4Y1, RPL24, RPS5 }\end{array}$ \\
\hline GO:0045653 negative & 7.26E-06 & HIST1H4L, HIST1H4B, HIST1H4E, HIST1H4C, HIST1H4D \\
\hline
\end{tabular}


megakaryocyte differentiation

GO:0034080 CENP-A containing nucleosome assembly

GO:0006412 translation

GO:0006342 chromatin silencing

GO:0006336 DNA replication-independent nucleosome assembly

GO:0006352 DNA-templated transcription, 1.26E-04 initiation

GO:1904837 beta-catenin-TCF

complex 2.55E-04 assembly

GO:0000028 ribosomal small subunit 3.25E-04 assembly

GO:0006123 mitochondrial electron transport, $3.80 \mathrm{E}-04$ cytochrome $\mathrm{c}$ to oxygen

GO:0045727 positive regulation of translation

GO:1902600 hydrogen ion transmembrane transport

GO:0043154 negative regulation of cysteinetype endopeptidase activity involved in apoptotic process

GO:0019731 antibacterial humoral response

3.90E-03

GO:0042274 ribosomal small subunit

biogenesis

GO:0002227 innate immune response in mucosa

GO:0050434 positive regulation of viral transcription

GO:0050830 defense response to Gram- 2.35E-02 positive bacterium

GO:0006368 transcription elongation from 2.42E-02

RNA polymerase II promoter

GO:0033489 cholesterol biosynthetic process

2.85E-02

via desmosterol

GO:0033490 cholesterol biosynthetic process

2.85E-02

via lathosterol

GO:0007568 aging

3.15E-02 CCL2, EEF2, SOD1, NQO1, APEX1

GO:0000302 response

to reactive oxygen

species
HIST1H4L, HIST1H4B, HIST1H4E, CENPW, HIST1H4C, HIST1H4D

RPS28, RPS29, RPL41, RPS15, RPL26, RPS13, RPS15A, RPS4Y1, RPL24, MRPS7, RPS5

HIST1H2AB, HIST2H2AB, HIST2H2AA4, HIST2H2AC, HIST1H2AE, HIST1H2AM

HIST1H4L, HIST1H4B, HIST1H4E, HIST1H4C, HIST1H4D

HIST1H4L, HIST1H4B, HIST1H4E, HIST1H4C, HIST1H4D

HIST1H4L, HIST1H4B, HIST1H4E, HIST1H4C, HIST1H4D

RPS28, RPS15, MRPS7, RPS5

COX7B, COX8A, COX6B1, COX6A1

EIF4G2, EEF2, QARS, PABPC1, THBS1

UQCR10, COX7B, COX8A, COX6B1, COX6A1

PRDX5, SFN, THBS1, IFI6, DHCR24

HIST1H2BK, HIST1H2BF, HIST1H2BI, B2M

RPS28, RRP36, RPS15

HIST1H2BK, HIST1H2BF, HIST1H2BI

POLR2L, POLR2K, SUPT4H1

HIST1H2BK, HIST1H2BF, HIST1H2BI, B2M

POLR2L, POLR2K, ELOF1, SUPT4H1

DHCR7, DHCR24

DHCR7, DHCR24

TXN, PRDX5, SOD1 


\begin{tabular}{lll}
\hline GO:0001895 retina homeostasis & $3.36 \mathrm{E}-02$ & ACTG1, SOD1, B2M \\
GO:0002576 platelet degranulation & $3.84 \mathrm{E}-02$ & PSAP, THBS1, SOD1, SRGN \\
GO:0043488 regulation of mRNA stability & $3.84 \mathrm{E}-02$ & PSMB3, HSPA1B, PABPC1, APEX1 \\
GO:0045471 response to ethanol & $4.03 \mathrm{E}-02$ & CCL2, EEF2, SOD1, NQO1 \\
GO:0006356 regulation of transcription from & $4.25 \mathrm{E}-02$ & POLR2L, POLR2K \\
RNA polymerase I promoter & & \\
GO:0098532 histone H3-K27 trimethylation & $4.25 \mathrm{E}-02$ & HIST1H1E, HIST1H1C \\
GO:0007596 blood coagulation & $4.41 \mathrm{E}-02$ & HIST1H3J, HIST1H3A, HIST1H3B, HIST1H3D, HIST1H3F \\
GO:0009615 response to virus & $4.52 \mathrm{E}-02$ & IFITM2, IFITM3, RPS15A, ENO1 \\
GO:0043066 negative regulation of apoptotic & $4.65 \mathrm{E}-02$ & KRT18, PLK2, PLK1, TMBIM4, PRDX5, THBS1, NQO1, DHCR24 \\
process & & \\
GO:1900121 negative regulation of receptor & $4.94 \mathrm{E}-02$ & ANXA2, B2M \\
binding & & \\
\hline \multicolumn{1}{c}{2} & &
\end{tabular}




\section{Table 5 (on next page)}

KEGG pathway enrichment analysis for DEGs in miRNA-mRNA regulatory network 
1 Table 5 KEGG pathway enrichment analysis for DEGs in miRNA-mRNA regulatory network

\begin{tabular}{|c|c|c|}
\hline Term & -value & Genes \\
\hline $\begin{array}{l}\text { hsa05322:Systemic } \\
\text { lupus erythematosus }\end{array}$ & 8 & $\begin{array}{l}\text { HIST1H2AB, HIST1H2BB, HIST1H3J, HIST1H4L, } \\
\text { HIST2H2AA4, HIST1H2BF, HIST1H2AE, } \\
\text { HIST1H2BO, HIST2H2AB, HIST1H2BK, HIST1H4B, } \\
\text { HIST2H2AC, HIST1H2BI, HIST1H3A, HIST1H4E, } \\
\text { HIST1H3B, HIST1H3D, HIST1H4C, HIST1H4D, } \\
\text { HIST1H3F, HIST1H2AM, HIST3H3 }\end{array}$ \\
\hline hsa05 & $2.00 \mathrm{E}-15$ & $\begin{array}{l}\text { HIST1H2AB, HIST1H2BB, HIST1H3J, HIST1H4L, } \\
\text { HIST2H2AA4, HIST1H2BF, HIST1H2AE, } \\
\text { HIST1H2BO, HIST2H2AB, HIST1H2BK, HIST1H4B, } \\
\text { HIST2H2AC, HIST1H2BI, HIST1H3A, HIST1H4E, } \\
\text { HIST1H3B, HIST1H3D, HIST1H4C, HIST1H4D, } \\
\text { HIST1H3F, HIST1H2AM, HIST3H3 }\end{array}$ \\
\hline hsa03 & 6.9 & $\begin{array}{l}\text { RPS28, RPS29, RPL41, RPS15, RPL26, RPS13, } \\
\text { RPS15A, RPS4Y1, RPL24, MRPS7, RPS5 }\end{array}$ \\
\hline $\begin{array}{l}\text { hsa05016:Huntington's } \\
\text { disease }\end{array}$ & 6.46 & $\begin{array}{l}\text { UQCR10, NDUFS5, POLR2L, POLR2K, COX7B, } \\
\text { COX8A, COX6B1, COX6A1, SOD1, ATP5H }\end{array}$ \\
\hline $\begin{array}{l}\text { hsa05203:Viral } \\
\text { carcinogenesis }\end{array}$ & $1.03 \mathrm{E}-03$ & $\begin{array}{l}\text { HIST1H2BO, HIST1H2BB, HIST1H4L, HIST1H2BK, } \\
\text { HIST1H2BF, HIST1H4B, HIST1H2BI, HIST1H4E, } \\
\text { HIST1H4C, HIST1H4D }\end{array}$ \\
\hline $\begin{array}{l}\text { hsa00190:Oxidative } \\
\text { phosphorylation }\end{array}$ & $1.36 \mathrm{E}-03$ & $\begin{array}{l}\text { UQCR10, NDUFS5, ATP6V0E1, COX7B, COX8A, } \\
\text { COX6B1, COX6A1, ATP5H }\end{array}$ \\
\hline $\begin{array}{l}\text { hsa05010:Alzheimer's } \\
\text { disease }\end{array}$ & $5.06 \mathrm{E}-03$ & $\begin{array}{l}\text { UQCR10, NDUFS5, COX7B, COX8A, COX6B1, } \\
\text { COX6A1, PSENEN, ATP5H }\end{array}$ \\
\hline $\begin{array}{l}\text { hsa05012:Parkinson's } \\
\text { disease }\end{array}$ & $8.75 \mathrm{E}-03$ & $\begin{array}{l}\text { UQCR10, NDUFS5, COX7B, COX8A, COX6B1, } \\
\text { COX6A1, ATP5H }\end{array}$ \\
\hline $\begin{array}{l}\text { hsa04260:Cardiac } \\
\text { muscle contraction }\end{array}$ & $1.4 J \mathrm{E}-02$ & UQCR10, COX7B, COX8A, COX6B1, COX6A1 \\
\hline $\begin{array}{l}\text { hsa04932:Non- } \\
\text { alcoholic fatty liver } \\
\text { disease (NAFLD) }\end{array}$ & 4.12E-02 & $\begin{array}{l}\text { UQCR10, NDUFS5, COX7B, COX8A, COX6B1, } \\
\text { COX6A1 }\end{array}$ \\
\hline
\end{tabular}

